\title{
UNA ESPECIE NUEVA DE LONGIOR TRAVASSOS ET KLOSS, 1958 (OXYURIDA: HYSTRIGNATHIDAE) PARÁSITA DE UN PASÁLIDO (COLEOPTERA: PASSALIDAE) DE PANAMÁ
}

\author{
Jans Morffe Rodríguez y Nayla García Rodríguez \\ Instituto de Ecología y Sistemática. Carretera de Varona km. 3²̌2, Capdevila, Boyeros, La Habana 19 \\ C.P.11900,Cuba.jans@ecologia.cu; nayla@ecologia.cu
}

\section{RESUMEN}

Se describe a Longior panamensis sp. nov. (Oxyurida: Hystrignathidae), parásito de un pasálido de Panamá. La especie es muy similar a L. elieri García, Ventosa et Morffe, 2009 (de Cuba) por la morfología del primer anillo cefálico, la presencia de anillos anchos en la cutícula cervical y las variables merísticas. Se diferencia por la extensión de las alas laterales y la forma del extremo terminal de las mismas. La cola de L. panamensis sp. nov. es, en proporción, algo más corta que en L. elieri y su anillo cefálico más ancho. Se enmienda la descripción de la extensión y la morfología de las alas laterales de L. elieri.

Palabras clave: Nematoda, Hystrignathidae, Longior, Passalidae, especie nueva, Panamá.

Title: Anew species of Longior Travassos et Kloss, 1958 (Oxyurida: Hystrignathidae) parasitizing a passalid beetle (Coleoptera: Passalidae) from Panama.

\section{ABSTRACT}

Longior panamensis sp. nov. (Oxyurida: Hystrignathidae) is described parasitizing a passalid beetle from Panama. It resembles L. elieri García, Ventosa et Morffe, 2009 (from Cuba) by having a similar morphology of the first cephalic annule, wide annule in the cervical cuticle and coincident meristic variables. They can be differentiated by the extension of the lateral alae as well as the form of the end of these structures. The tail of L. panamensis $\mathrm{sp}$. nov. is comparatively shorter and its first cephalic annule wider. Amendments to the description of the extension and morphology of the lateral alae of L. elieri are presented.

Key words: Nematoda, Hystrignathidae, Longior, Passalidae, new species, Panama.

\section{INTRODUCCIÓN}

El género Longior Travassos et Kloss, 1958, agrupa a aquellas especies de nemátodos parásitos del intestino de coleópteros pasálidos, cuyas hembras presentan el cuerpo esofágico subcilíndrico, cutícula cervical inerme, sistema reproductor monodelfo-prodelfo y huevos provistos de estrías rugosas longitudinales (Adamson y Van Waerebeke, 1992). Hasta el presente se han descrito seis especies, de las cuales cuatro se distribuyen en Cuba: L. alius García y Coy, 1994; L. elieri García, Ventosa et Morffe, 2009; L. longior Morffe et García, 2011 y L. similis Morffe, García et Ventosa, 2009 (García y Coy, 1994; García et al., 2009; Morffe et al., 2009; Morffe y García, 2011). Las restantes especies son L. longicollis (Artigas, 1926), de Brasil (especie tipo del género) y L. semialata Hunt, 1981 de Santa Lucía, Antillas Menores (Hunt, 1981; Travassos y Kloss, 1958). La familia Hystrignathidae ha sido poco estudiada en Panamá, a pesar de la gran diversidad de pasálidos presente en la región. Morffe y García (2010) describieron a Hystrignathus dearmasi Morffe et García, 2010, el único histrignátido panameño registrado hasta el momento. 


\title{
OBJETIVO
}

- Describir una especie nueva de Longior de Panamá.

\section{MATERIALES Y MÉTODOS}

Dos especímenes de un pasálido no identificado fueron recolectados manualmente en troncos podridos del Parque Nacional "Summit", Panamá. Los hospederos fueron sacrificados por decapitación, los intestinos extraídos inmediatamente y fijados en etanol al 70\%. Los mismos fueron disecados en placas Petri con el propio líquido fijador, con ayuda de pinzas de punta fina y agujas enmangadas. Los parásitos hallados se extrajeron y conservaron en etanol $70 \%$.

Los nemátodos se transfirieron gradualmente a glicerina anhidra y se montaron de forma permanente en el mismo medio. Los bordes de los cubreobjetos se sellaron con esmalte de uñas para evitar la hidratación de la glicerina. Las medidas se tomaron con ayuda de un micrómetro ocular, siguiendo el criterio de Morffe y García (2010) y están dadas en milímetros. Se calcularon los índices de De Man a, b, c y V\%. De cada variable se expresa su rango, seguido por la media y la desviación estándar entre paréntesis, así como el número de mediciones. Se revisó además el material tipo de L. elieri depositado en la Colección Helmintológica de las Colecciones Zoológicas (CZACC) del Instituto de Ecología y Sistemática, La Habana, Cuba.

Las microfotografías se obtuvieron con ayuda de una cámara digital AxioCam, acoplada a un microscopio Carl Zeiss AxioScop 2 Plus. Los dibujos fueron realizados con los programas CorelDRAW X3 y Adobe Photoshop CS2, sobre la base de las microfotografías. Las escalas de todas las ilustraciones están dadas en milímetros.

El material tipo está depositado en la Colección Helmintológica de las Colecciones Zoológicas (CZACC) del Instituto de Ecología y Sistemática, La Habana, Cuba y en la Colección Helmintológica del Instituto Oswaldo Cruz (CHIOC), Río de Janeiro, Brasil.

\author{
RESULTADOS \\ TAXONOMÍA \\ Hystrignathidae \\ Longior Travassos et Kloss, 1958
}

Longior panamensis sp. nov.

Figuras 1, A-G; 2, A-D; 3B

Diagnosis. Hembras con el primer anillo cefálico apenas dilatado. Cutícula cervical provista de anillos anchos. Poro excretor muy próximo al bulbo esofágico. Alas laterales que se extienden ligeramente más allá del nivel del ano, formando un ángulo agudo con el eje del cuerpo en su extremo terminal.

Diagnosis. Females with the first cephalic annule barely inflated. Cervical cuticle with wide annule. Excretory pore very close to the basal bulb. Lateral alae extending to a short distance beyond the level of the anus. The margins of the lateral alae form sharp angles with the body axis in their terminal ends.

Descripción. Hembras con el cuerpo largo y relativamente delgado. Cutícula cervical inerme, provista de anillos anchos (cerca de $10 \mu \mathrm{m})$, que se vuelven más estrechos $(7 \mu \mathrm{m})$ a continuación del nivel del anillo nervioso. 

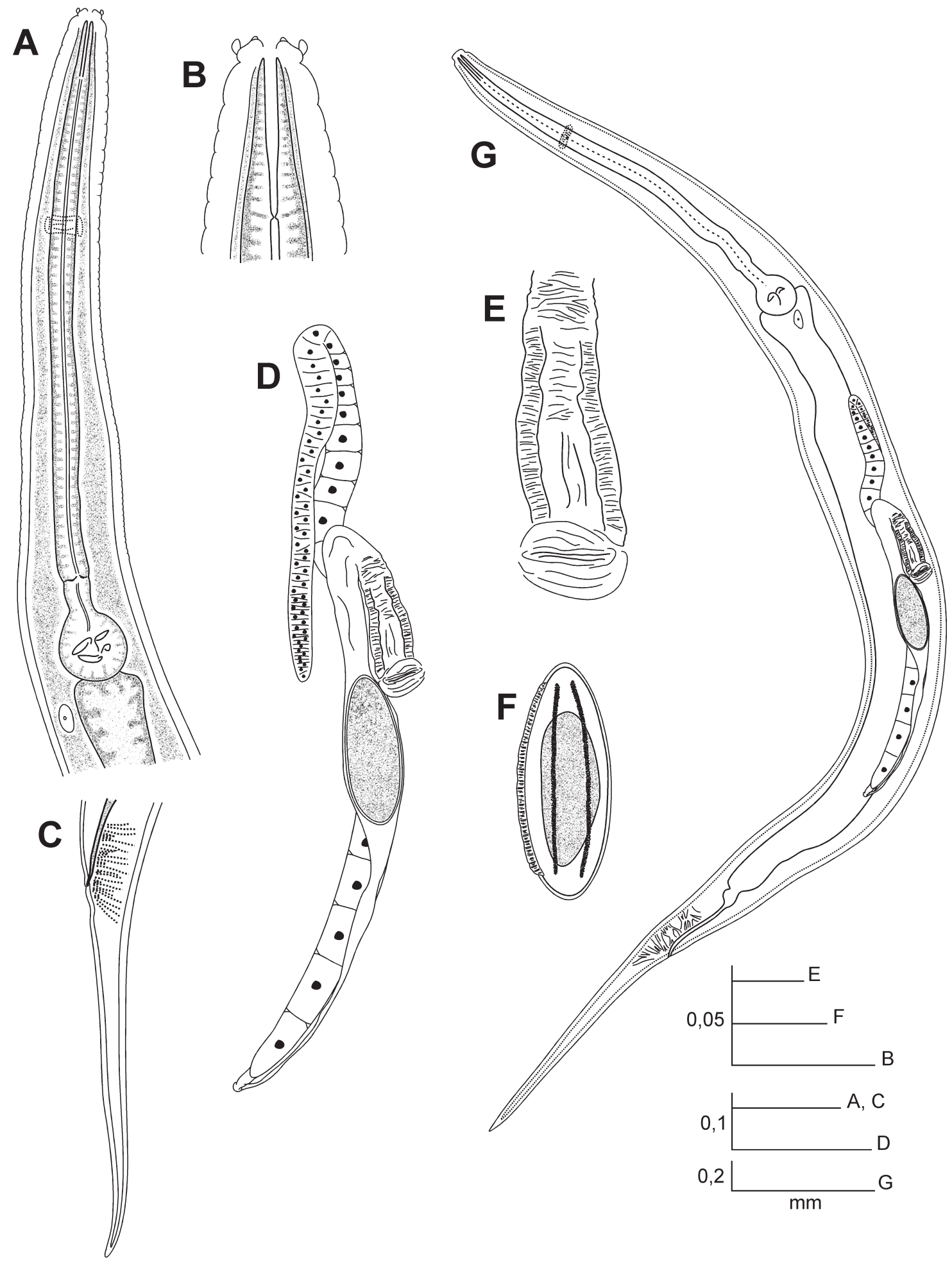

Figura 1. Longior panamensis sp. nov., hembra. A, región esofágica, vista ventrolateral. B, extremo cefálico. C, cola, vista lateral. $\mathrm{D}$, sistema reproductor, vista ventrolateral. E, vulva, vista ventral. F, huevo. G, habitus, vista ventral. 

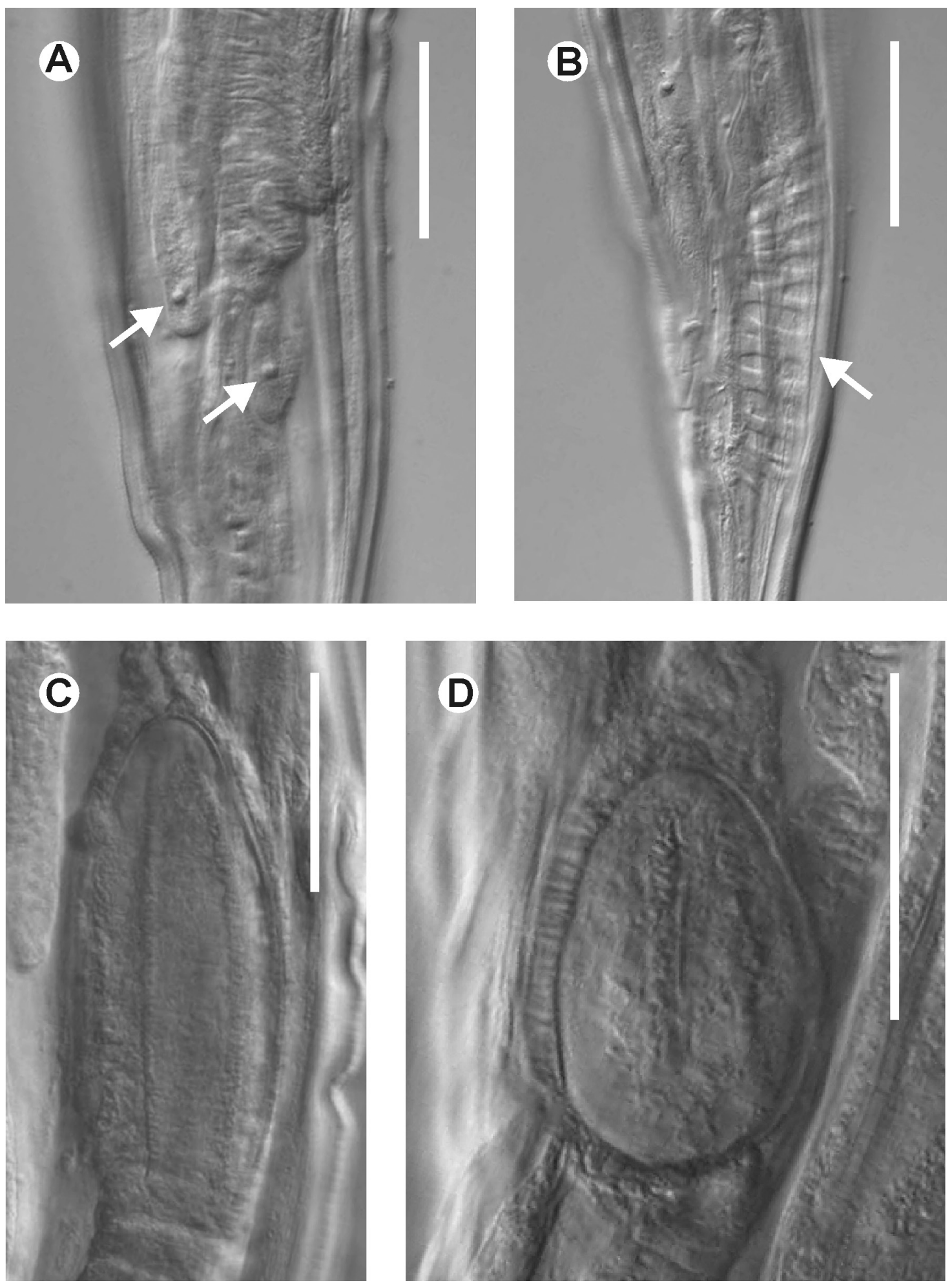

Figura 2. Longior panamensis sp. nov., hembra. A, glándulas rectales (señaladas por las flechas). B, región del ano mostrando los haces musculares asociados (señalado por la flecha). C, huevo. D, huevo atrofiado hallado en uno de los parátipos. Líneas de escala: $0.05 \mathrm{~mm}$. 

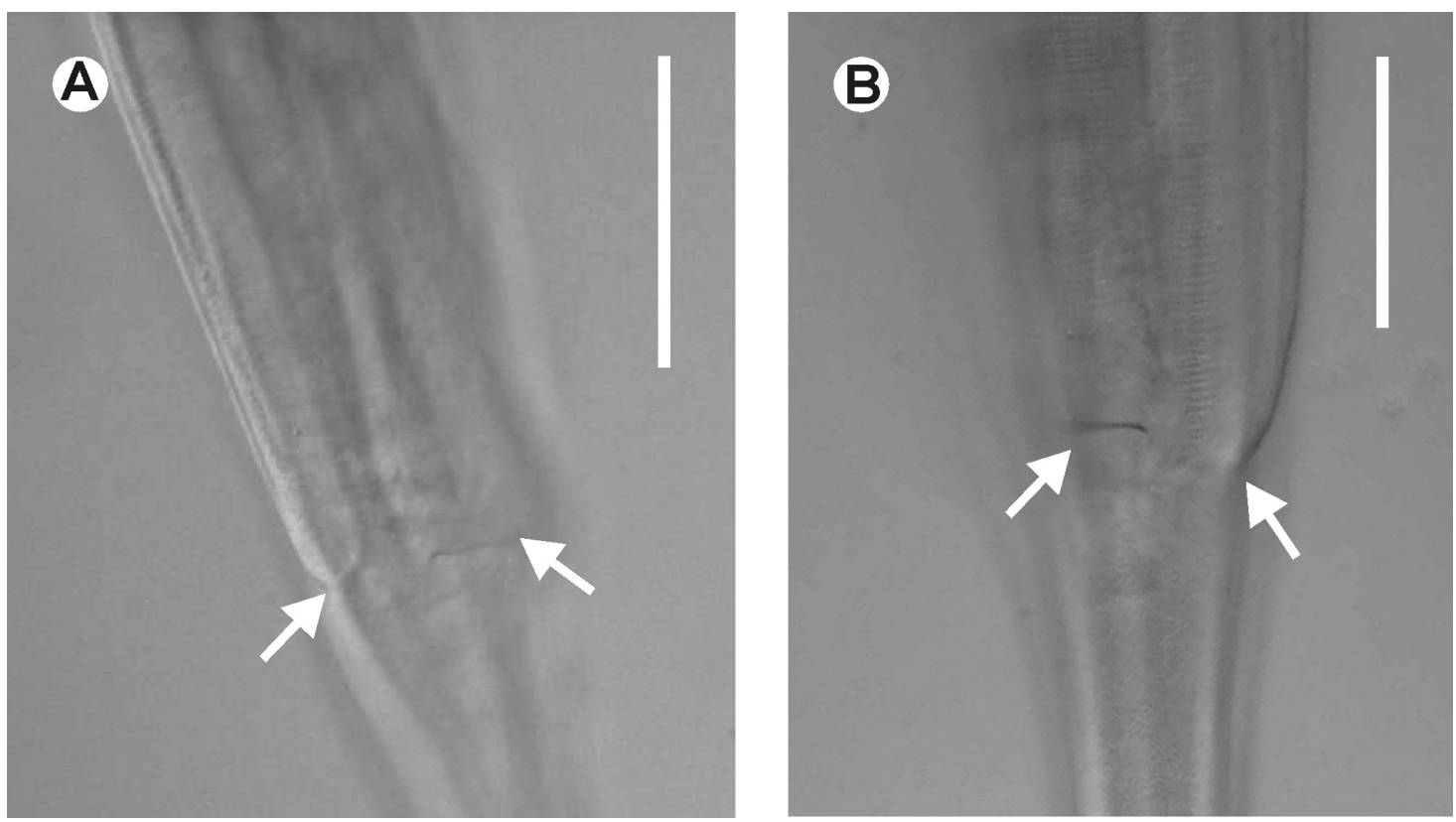

Figura 3. Región final de las alas laterales en las hembras de Longior elieri García, Ventosa et Morffe, 2009 y L. panamensis sp. nov. (final de las alas y ano señalados por las flechas). A, L. elieri. B, L. panamensis sp. nov. Líneas de escala: $0.05 \mathrm{~mm}$.

Tras el final del cuerpo esofágico y hasta el nivel del ano los anillos se vuelven muy estrechos (cerca de $1 \mu \mathrm{m}$ ) y apenas visibles. Estrías subcuticulares longitudinales presentes. Alas laterales desarrolladas que surgen a cierta distancia de la base del bulbo esofágico (aproximadamente el doble del ancho máximo del cuerpo) y terminan detrás del nivel del ano. El margen de las alas laterales, en su región terminal forma un ángulo agudo con el eje longitudinal del cuerpo. Anillo labial separado del cuerpo por un surco simple y provisto de ocho papilas grandes y pareadas. Primer anillo cefálico cónico, apenas dilatado, de cerca del doble de la longitud del anillo labial. Estoma largo (de cerca de 3.5 veces la longitud del primer anillo cefálico), rodeado por un collar esofágico. Esófago con un cuerpo esofágico muscular, subcilíndrico, muy elongado y con la base ligeramente diferenciada del istmo. Bulbo esofágico subesférico, con válvulas bien desarrolladas. Intestino simple, subrectilíneo, con la región anterior ligeramente dilatada. Recto largo y ano no prominente. Al menos dos glándulas rectales visibles, de forma elíptica. Anillo nervioso rodeando al cuerpo esofágico, situado aproximadamente al $30 \%$ de su longitud. Poro excretor postbulbar, separado del bulbo por una distancia equivalente a un quinto del ancho máximo del cuerpo. Vulva en forma de ranura media transversal, cerca de la mitad del cuerpo, labios no prominentes. Vagina musculosa, bien desarrollada y dirigida hacia la región anterior. Sistema reproductor monodelfo-prodelfo. Ovario reflexo, flexión distal equivalente al doble del ancho máximo del cuerpo. Huevos ovoidales, con ocho crestas rugosas longitudinales que no llegan a los polos. Ovocitos dispuestos en una sola hilera. Cola cónica, atenuada y terminada en una punta fina. Macho desconocido.

Tipos. Holótipo (hembra), CZACC 11.4646, en Passalidae no identificado; Parque Nacional "Summit", Provincia Panamá, Panamá; 25/IX/2009; L. F. de Armas col. Parátipos (6 hembras), CZACC 11.4647-11.4652, idénticos datos que el holótipo. Parátipos (2 hembras), CHIOC, idénticos datos que los anteriores.

Otro material examinado. Holótipo (hembra) de L. elieri; CZACC 11.4418, en Passalus pertyi; Sierra de Casas, Isla de La Juventud, Cuba; IX/2004; E: Fonseca y D. Ortíz col. Parátipos (3 hembras) de L. elieri, CZACC 11.4419-11.4421, idénticos datos que el holótipo. 
Medidas. Holótipo (hembra), $\mathrm{a}=17.91 ; \mathrm{b}=3.65 ; \mathrm{c}=5.47 ; \mathrm{V} \%=49.75$; longitud total $=1.970$; ancho máximo $=0.110$; anillo cefálico $($ largo $\times$ ancho $)=0.013 \times 0.033$; longitud del estoma $=$ 0.053 ; longitud del cuerpo esofágico $=0.440$; longitud del istmo $=0.033$; diámetro del bulbo esofágico $=0.060$; longitud total del esófago $=0.540$; distancia del anillo nervioso al extremo anterior $=0.185$; distancia del poro excretor al extremo anterior $=0.600$; distancia de la vulva al extremo posterior $=0.990$; longitud de la cola $=0.360$; huevos $=0.120 \times 0.045(\mathrm{n}=1)$.

Parátipos hembras $(\mathrm{n}=8), \mathrm{a}=13.36-17.90(15.40 \pm 1.52, \mathrm{n}=7) ; \mathrm{b}=3.59-4.20(3.82 \pm 0.19$, $\mathrm{n}=7) ; \mathrm{c}=5.42-6.30(5.85 \pm 0.28, \mathrm{n}=8) ; \mathrm{V} \%=50.28-51.91(51.03 \pm 0.70, \mathrm{n}=6)$; longitud total = 1.670-2.060 $(1.864 \pm 0.123, \mathrm{n}=8)$; ancho máximo $=0.100-0.140(0.121 \pm 0.013, \mathrm{n}=7)$; anillo cefálico $($ largo $\times$ ancho $)=0.013 \times 0.033(\mathrm{n}=1)$; longitud del estoma $=0.045-0.060(0.053$ $\pm 0.005, \mathrm{n}=8)$; longitud del cuerpo esofágico $=0.370-0.450(0.410 \pm 0.029, \mathrm{n}=7)$; longitud del istmo $=0.025-0.033(0.028 \pm 0.003, \mathrm{n}=7)$; diámetro del bulbo esofágico $=0.053-0.068(0.061 \pm$ $0.006, \mathrm{n}=8)$; longitud total del esófago $=0.450-0.550(0.491 \pm 0.040, \mathrm{n}=7)$; distancia del anillo nervioso al extremo anterior $=0.178-0.198(0.186 \pm 0.008, \mathrm{n}=8)$; distancia del poro excretor al extremo anterior $=0.540-0.650(0.580 \pm 0.048, \mathrm{n}=4)$; distancia de la vulva al extremo posterior $=0.880-1.020(0.918 \pm 0.053, \mathrm{n}=6)$; longitud de la cola $=0.290-0.340(0.319 \pm 0.019)$; huevos $=0.110-0.123 \times 0.038-0.053(0.118 \pm 0.003 \times 0.045 \pm 0.004, \mathrm{n}=10)$.

Hospedero tipo. Passalidae no identificado.

Localización. Ciegos del intestino posterior.

Etimología. Epíteto específico referido al país donde se ubica la localidad tipo de la especie.

Tabla 1. Medidas (expresadas en milímetros) de las hembras de Longior panamensis sp. nov. y Longior elieri García, Ventosa et Morffe, 2009 (Nematoda: Hystrignathidae) procedentes del Parque Nacional "Summit", Panamá y Sierra de Casas, Isla de La Juventud, Cuba, respectivamente.

\begin{tabular}{|l|l|l|}
\hline Especie & L. panamensis sp. nov. & L.elieri \\
\hline Localidad & $\begin{array}{l}\text { Parque Nacional “Summit”, Panamá } \\
\mathrm{n}=9\end{array}$ & $\begin{array}{l}\text { Sierra de Casas, Isla de La Juventud, } \\
\text { Cuba } \\
\text { (localidad tipo) } \\
\mathrm{n}=4\end{array}$ \\
\hline Hospedero & Passalidae no identificado & Passalus pertyi \\
\hline Longitud total & $1.670-2.060$ & $1.650-1.950$ \\
\hline Ancho máximo & $0.100-0.140$ & $0.100-0.120$ \\
\hline Anillo cefálico (largo $\times$ ancho) & $0.013 \times 0.033$ & $0.013-0.015 \times 0.025$ \\
\hline Longitud del estoma & $0.045-0.060$ & $0.050-0.055$ \\
\hline Longitud del cuerpo esofágico & $0.370-0.450$ & $0.360-0.450$ \\
\hline Longitud del istmo & $0.025-0.033$ & $0.025-0.030$ \\
\hline Diámetro del bulbo esofágico & $0.053-0.068$ & $0.058-0.068$ \\
\hline Longitud total del esófago & $0.450-0.550$ & $0.455-0.490$ \\
\hline $\begin{array}{l}\text { Distancia anillo nervioso-extremo } \\
\text { anterior }\end{array}$ & $0.178-0.198$ & $0.173-0.180$ \\
\hline $\begin{array}{l}\text { Distancia poro excretor-extremo } \\
\text { anterior }\end{array}$ & $0.540-0.650$ & $0.520-0.550$ \\
\hline Distancia vulva-extremo posterior & $0.880-1.020$ & $0.960-1.050$ \\
\hline Longitud de la cola & $0.290-0.360$ & $0.330-0.390$ \\
\hline Huevos & $0.110-0.123 \times 0.038-0.053$ & $0.115-0.123 \times 0.045-0.048$ \\
\hline a & $13.36-17.91$ & $17.11-18.44$ \\
\hline b & $3.59-4.20$ & $3.65-3.98$ \\
\hline c & $5.42-6.30$ & $5.00-5.34$ \\
\hline V\% & $50.28-51.91$ & 50.77 \\
\hline
\end{tabular}




\section{DISCUSIÓN}

L. panamensis sp. nov. es muy similar a L. elieri, por tener las hembras el primer anillo cefálico apenas dilatado, la cutícula cervical provista de anillos anchos y el poro excretor muy próximo al bulbo esofágico. La mayoría de las variables morfométricas también coinciden en ambas especies (Tabla 1). Sin embargo, puede diferenciarse del taxon cubano por presentar las alas laterales que se extienden ligeramente más allá del nivel del ano. En su extremo terminal, dichas alas laterales forman un ángulo agudo con el eje del cuerpo, como ocurre en la mayoría de los histrignátidos, mientras que L. elieri posee alas laterales que terminan en el nivel del ano y forman un lóbulo redondeado en ese punto. La extensión de las alas laterales y la forma de su extremo terminal fueron observados en la revisión del material tipo de L. elieri y no aparecen en la descripción original de la especie (García et al., 2009). L. panamensis sp. nov. también difiere de $L$. elieri en la longitud de la cola, en proporción más corta $(c=5.42-6.30$ vs. 5.00-5.34) y porque el primer anillo cefálico es más ancho (0.033 vs. 0.025).

L. similis también presenta la cutícula cervical con anillos marcados, pero puede distinguirse de L. panamensis sp. nov. por la mayor dilatación del primer anillo cefálico (Morffe et al., 2009). Además, en L. similis el poro excretor es más posterior, situado a una distancia del bulbo esofágico equivalente al ancho del cuerpo. En L. panamensis sp. nov. el poro está más próximo al bulbo, a una distancia de aproximadamente un quinto del ancho del cuerpo.

L. semialata y L. alius se diferencian de L. panamensis sp. nov. por la menor extensión de las alas laterales. En el primero, estas van desde el nivel de la vulva hasta justo antes del nivel del ano (Hunt, 1981) y en el segundo se extienden desde el punto medio entre el ano y la vulva hasta el propio ano. L. alius presenta, además, el istmo más largo y plegado (García y Coy, 1994).

L. longior es la especie de mayor talla del género y difiere notablemente por la longitud total (3.500-4.530 vs. 1.670-2.060) del cuerpo. El primer anillo cefálico está mucho más dilatado en L. longior, el cual, además, carece de anillos marcados en la cutícula cervical (Morffe y García, 2011). L. longicollis puede separarse por su mayor tamaño (2.980-3.640 vs. 1.670-2.060) y por presentar el esófago $(\mathrm{b}=4.79-4.97$ vs. 3.59-4.20) y la cola $(\mathrm{c}=9.03-9.33$ vs. 5.42-6.30), en proporción, más cortos.

\section{AGRADECIMIENTOS}

Los autores desean agradecer al Dr. Luis F. de Armas, del Instituto de Ecología y Sistemática, por recolectar los hospederos. Al MSc. Eduardo Furrazola (Instituto de Ecología y Sistemática) por su ayuda con las microfotografías. A IDEAWILD por su donativo para el trabajo de laboratorio. Este trabajo está inscrito en el proyecto "Colecciones Zoológicas, su Conservación y Manejo" del Programa Ramal de Ciencia y Técnica de Diversidad Biológica del Ministerio de Ciencia, Tecnología y Medio Ambiente de Cuba.

\section{LITERATURA CITADA}

Adamson, M. L. y D. Van Waerebeke. 1992. Revision of the Thelastomatoidea, Oxyurida of invertebrate hosts III. Hystrignathidae. Systematic Parasitology, 22: 111-130.

García, N. y A. Coy. 1994. Descripción de dos nuevas especies y registro de nuevos hospederos de nemátodos (Nematoda) de la región oriental de Cuba. Avicennia, 1: 13-17.

García, N., L. Ventosa y J. Morffe. 2009. Dos especies nuevas de los géneros Lepidonema y Longior (Thelastomatoidea: Hystrignathidae) de la Isla de la Juventud, Cuba. Solenodon, 8: $1-7$. 
Hunt, D. J. 1981. On Artigasia horridospina n. sp., Longior semialata n. sp., Mentecle magnifica n. sp., Paraxyo ensicrinatus n. sp. (Oxyurida: Hystrignathidae) and Pulchrocephala? pulchrocephala Travassos, 1925 (Oxyurida: Pulchrocephalidae). Systematic Parasitology, 3: 33-52.

Morffe, J. y N. García. 2010. Hystrignathus dearmasi sp. n. (Oxyurida, Hystrignathidae), first record of a nematode parasitizing a Panamanian Passalidae (Insecta, Coleoptera). ZooKeys, 57: 1-8.

Morffe, J. y N. García. 2011. On some new species of the genus Longior Travassos \& Kloss, 1958 (Oxyurida, Hystrignathidae) with description of a new species. ZooKeys, 78: 1-14.

Morffe, J., N. García y L. Ventosa. 2009. Longior similis sp. nov. (Thelastomatoidea: Hystrignathidae) parasite of Passalus interstitialis from western Cuba and new records of Longior zayasi. Solenodon, 8: 12-19.

Travassos, L. y G. R. Kloss. 1958. Sobre a fauna dos nematodeos dos Coleopteros Passalidae da Estaçao Biologica de Boraceia. Arquivos de Zoologia do Estado de Sao Paulo, 11(2): 23-57.

[Recibido: 31 de enero, 2012. Aceptado para publicación: 13 de marzo, 2012] 